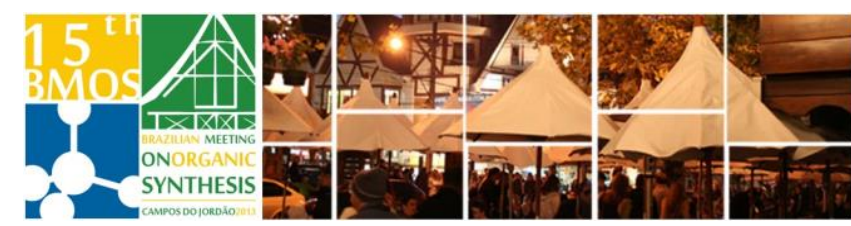

\title{
Asymmetric Ring Contraction Reactions Mediated by Chiral I(III)
}

\author{
Anees Ahmad and Luiz F. Silva, Jr.* \\ Instituto de Química - Universidade de São Paulo, Av. Prof. Lineu Prestes, 748, CP 26077, CEP 05513-970 \\ São Paulo SP, Brazil. \\ anees_chemist@yahoo.com, luizfsjr@iq.usp.br.*
}

Keywords: Hypervalent lodine, Rearrangement, Ring Contraction.

\section{INTRODUCTION}

Hypervalent iodine reagents are widely used in chemical synthesis. ${ }^{1}$ The development of reaction processes controlled by chiral hypervalent iodine reagents with high enantioselectivity has drawn extensive and enduring attention because of their environmentally friendly profile and avoids the issue of toxicity. ${ }^{2,3}$ Rearrangement reaction is one of the important reactions promoted by $\mathrm{I}(\mathrm{III}){ }^{1,3,4}$ Herein, we describe asymmetric ring contraction reactions mediated by chiral hypervalent iodine reagents.

\section{RESULTS AND DISCUSSION}

Optically active aryl iodide reagents (1-5) were prepared according to reported protocol (Scheme 1). ${ }^{3}$

1 $y_{1}{ }^{\circ}$

Scheme 1. Optically active chiral aryl iodide reagents

First step, koser's derivative chiral aryl iodine(III) reagents were generated in situ by treating chiral iodobenzene with $m C P B A$ and $\mathrm{TsOH}_{2} \mathrm{O}$ at room temperature using appropriate solvents. Second step involve the addition of small amount of water (22 equiv), followed by addition of alkene 6 . The aldehyde formed in this process was reduced in situ adding $\mathrm{NaBH}_{4}$. lodide 2 and 4 afforded alcohol 7 as non-racemic mixture using $\mathrm{CF}_{3} \mathrm{CH}_{2} \mathrm{OH}$ (TFE)/DCM as mixed solvent (entries 4 and 6, Table 1).

Table 1. Asymmetric ring contraction of alkene 6 with chiral I(III)

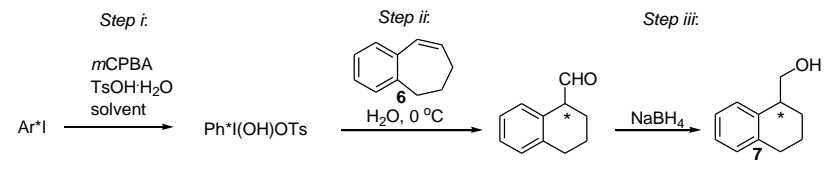

\begin{tabular}{|c|c|c|c|}
\hline Entry & Conditions & Yield & $e e$ \\
\hline 1 & 1,HFP/DCM(1:6) & 20 & 7 \\
\hline 2 & 2,HFP/DCM(1:6) & $28(49 S M)$ & 8 \\
\hline 3 & 2, TFEDCM (1:4) & $42(40 S M)$ & 1 \\
\hline 4 & 2, TFEDCM (1:1) & $28(39 S M)$ & 13 \\
\hline 6 & 3,HFP/DCM $(1: 6)$ & SM & - \\
\hline 7 & 3,TFE/DCM $(1: 1)$ & SM & - \\
\hline 5 & 4,HFIP/DCM $(1.6)$ & - & 3.5 \\
\hline 6 & 4, TFE/DCM(1:1) withoutPTSA & - & 16 \\
\hline
\end{tabular}

The use of two arms chiral aryl iodide reagents like $\mathbf{4}$ and $\mathbf{5}$, using mixed solvent system $\left(\left(\mathrm{CF}_{3}\right)_{2} \mathrm{CHOH}(\mathrm{HFIP}) / \mathrm{CH}_{2} \mathrm{Cl}_{2}\right)$, gave corresponding indane 9 in $15 \%$ and $20 \%$ ee, respectively (entries 3 and 7 , Table 2). A slight increase in ee was observed when (+) camphorsulfonic acid (CSA) was used instead of PTSA (entry 6).

Table 2. Asymmetric ring contraction of alkene 8 with chiral I(III)

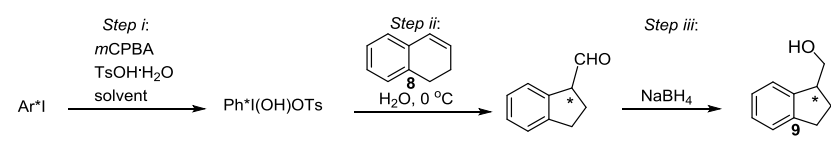

\begin{tabular}{|c|c|c|c|}
\hline Entry & Conditions & Yield & $e$ \\
\hline 1 & 2,HFIP/DCM(1:6) & - & 7 \\
\hline 2 & 2, TFE/DCM(1:1) & - & 6 \\
\hline 3 & 4,HFIP/DCM(1:6) & - & 15 \\
\hline 4 & 4, TFEDCM (1:1) & 59 (25SM) & 5 \\
\hline 5 & 4,TFE/DCM(1:1) withoutPTSA & - & 4 \\
\hline 6 & 4, HFIP/DCM (1:6) with (+)CSA & $37(11 \mathrm{SM})$ & 25 \\
\hline 7 & 5,HFIP/DCM $(1: 6)$ & 57 (28SM) & 20 \\
\hline 8 & 5, TFE/DCM(1:1) & $-(66 \mathrm{SM})$ & 6 \\
\hline
\end{tabular}

Chromane 11 was obtained in $18 \%$ and $19 \%$ ee using iodide 4 (entries 4 and 5, Table 3). However, when aryl iodide 4 was used without adding PTSA, desired alcohol was obtained in $23 \%$ ee (entry 6 ).

Table 3. Asymmetric ring contraction of alkene $\mathbf{1 0}$ with chiral I(III)

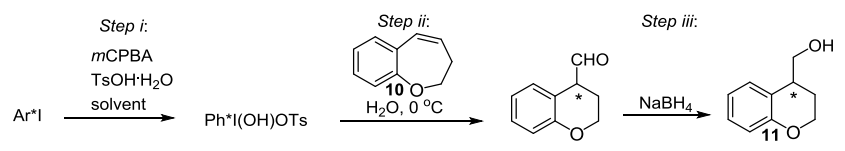

\begin{tabular}{|l|l|l|l|}
\hline Entry & Conditions & Yield\% & ee\% \\
\hline 1 & 2,HFIP/DCM(1:6) & - & 13 \\
\hline 2 & 2, TFE/DCM $(1: 4)$ & - & 3 \\
\hline 3 & 2, TFE/DCM $(1: 1)$ & - & 6 \\
\hline 4 & 4,HFIP/DCM(1:6) & $54(13 S M)$ & 18.5 \\
\hline 5 & 4, TFE/DCM $(1: 1)$ & $33(25 S m)$ & 19 \\
\hline 6 & 4, TFE/DCM (1:1) withoutPTSA & - & 23 \\
\hline
\end{tabular}

CONCLUSION

The reactivity of several chiral aryl iodine(III) reagents with benzo-fused cyclic alkenes was examined. Studies to get high ee and yield are underway.

\section{ACKNOWLEDGEMENTS}

Thanks CAPES, FAPESP and CNPq for financial support.

\section{REFERENCES}

${ }^{1}$ Silva, L. F., Jr.; Olofsson, B. Nat. Prod. Rep. 2011, 28, 1722. ${ }^{2}$ Liang, H.; Ciufolini, M. A. Angew. Chem. Int. Ed. 2011, 50, 11849. ${ }^{3}$ Farid, U.; Malmedy, F.; Claveau, R.; Albers, L.; Wirth, T. Angew. Chem. Int. Ed. 2013, 52, 7018. ${ }^{4}$ Silva, L. F., Jr.; Siqueira, F. A.; Pedrozo, E. C.; Vieira, F. Y. M.; Doriguetto, A. C. Org. Lett. 2007, 9, 1433. 